\title{
Le rôle de l'Espagne dans la transmission de l'iconographie biblique *
}

\author{
Gabrielle Sed-Rajna **
}

Deux facteurs d'importance fondamentale ont contribué à l'essor des recherches sur l'iconographie biblique et ses origines durant ces dernières décennies. Le fait majeur, lequel a véritablemente bouleversé toutes les théories antérieures, fut la découverte, en 1932, des peintures murales de la synagogue de Doura-Europos. Le second facteur était l'elaboration d'une méthode nouvelle pour l'analyse des images, méthode conçue par Kurt Weitzmann et développée par ses disciples de Princeton.

Pour la commodité de l'exposé, nous tenterons de caractériser d'abord cette nouvelle méthode dont l'application aux recherches sur l'imagerie biblique a été particulièrement riche en résultats ${ }^{1}$. L'essentiel de la méthode est de considérer l'iconographie comme un langage, dont les formules, les composantes, sont des éléments autonomes tout comme les mots et la syntaxe qui composent le langage écrit. L'analyse de ces composantes permet non seulement de comprendre le sens véritable des images, mais également de retracer leur histoire, de retrouver la filiation de leur transmission, voir de leur «migration». En effet, certaines formules, créées pour un texte défini, ont pu être transférées pour visualiser un récit différent. Un exemple souvent cité, et qui illustre clairement ce procédé, est l'iconographie de Samson maîtrisant le lion, représenté par une formule déjà employée pour l'illustration de Hercule combattant le lion de Numéa. En d'autres exemples la formule iconographique, conçue pour illustrer un texte, fut reprise dans des copies plus tardives du même texte, mais avec

* Ponencia presentada en el Encuentro Internacional de Historiadores En torno a Sefarad. Toledo, 1991.

** Directeur de recherche au CNRS PARIS.

1 Cf. Weitzmann, K., lliustrations in Roll and Codex. Princeton 1970. 
des modifications qui reflètent les changements intervenus dans l'interprétation du texte. De telles modifications, nous en verrons des exemples plus tard, sont révélateurs des tendances intellectuelles d'une époque ou d'un milieu. Par ailleurs, des cycles d'images, qui accompagnaient un texte, pouvaient servir de répertoire à l'illustration d'autres œuvres littéraires. Tel est le cas des cycles d'images créés pour l'illustration des bibles, dont certains éléments furent transférés pour illustrer les Evangiles, les rituels, ou des élaborations historiques. L'analyse des formules permet souvent de retracer leur cheminement et même de retrouver éventuellement le contexte originel pour lequel elles avaient été créées. Reprendre toutes les données acquises grâce à cette méthodologie, dépasserait le cadre fixé à cet exposé. Nous en retiendrons ici seulement ces quelques principes directeurs, nécessaires pour la suite de notre propos.

Les innombrables études consacrées aux peintures de Doura-Europos ${ }^{2}$ nous dispensent d'entrer dans les détails. Rappellons simplement les principaux points acquis:

1) L'ensemble des peintures narratives de la deuxième phase de la synagogue constitue une composition programmée pour exprimer l'attente messianique, l'espoir de la délivrance ayant été aiguisé par l'actualité politique de Doura, qui vécut sous la menace des Parthes. La composition reflète également un agencement à fonction liturgique ${ }^{3}$, les deux interprétations pouvant d'ailleurs être conjuguées.

2) Les panneaux qui composent l'ensemble sont construits à partir d'épisodes narratifs. Si la sélection des épisodes était subordonnée au programme idéologique, les épisodes eux-mêmes sont purement descriptifs. La structure des panneaux, la coordination des scènes individuelles, attestant des suppressions de maillons intermédiaires ou, au contraire, des répétitions des protagonistes aux phases successives du récit, sont des indices qui invitent à supposer que les peintres, ou ceux qui ont conçu les peintures, disposaient de répertoires de scènes narratives détaillés dont les réalisateurs de Doura ont retenu les éléments qui servaient à leur objectif. Ils les organisaient, par «condensation», par conjugaison ou par juxtaposition, en foction des possibilités spaciales de la salle et en foction des nécessités du programme. Cette supposition entraine inévitablement

\footnotetext{
Kraeling, C.H., The Excavations at Dura Europos. Final Report. VIII. The Synagogue. NewHaven 1956 et la bibliographie ibidem, WeITZMANN, K., KeSSLER, H.L. The Frescoes of the Dura Synagogue and Christian Art. Washington 1990.

3 Cf. GutmanN, J., "Programmatic Painting in Dura", dans The Dura-Europos Synagogue: a Re-evaluation (1932-1972). Missoula Montana 1973, 130-150.
} 
celle de l'existence d'une imagerie fondée sur le texte biblique, de caractère descriptif et purement narratif, conçue et diffusée avant l'achèvement des peintures en 245 de notre ère. Faute de preuve archéologique, on ignore sous quelle forme une telle imagerie a pu être réalisée, l'hypothèse la plus probable étant celle d'illustrations accompagnant un texte.

3) Il a été démontré que les peintures de Doura illustrent un texte biblique enrichi d'éléments interprétatifs dont les sources sont les bibles historiées de la littérature rabbinique. Ces textes sont connus aujourd'hui à travers des compilations parfois tardives - le Targum, la littérature apocryphe- dont les originaux ont été écrits entre $1^{e}-\left.1\right|^{e}$ siécle avant et le $\| l^{e}$ $1 I^{\ominus}$ siècle après J.C., probablement en Palestine. L'époque correspond bien à celle pendant laquelle des versions illustrées de ces bibles historiées devaient être créées, et plusieurs indices font penser que la Palestine était le lieu d'origine de ces dernières également. Ce qui est certain, c'est que la forme littéraire et la version visuelle de ces bibles historiées reflètent la même mentalité, si typique de la littérature rabbinique: le souci de fidélité au texte, la recherche du détail, la volonté de faire revivre le récit dont le moindre élément est considéré comme signifiant.

4) Si l'on est réduit à des hypothèses en ce qui concerne les modèles de Doura, des indices plus nombreux et plus claires prouvent que des éléments de cette iconographie, voire quelques compositions, ont été repris au cours des siècles en d'autres œuvres. Toutefois, lorsqu'on évoque Doura comme source d'œuvres plus récentes, lorsqu'en retraçant la filiation de certains thèmes on remonte jusqu'aux peintures de la synagogue, il y a un point essentiel qu'on ne soulignera jamais assez: les peintures elles-mêmes n'ont pu servir de modèle à aucune œuvre. La salle de prière était visible de 245 , date de l'achèvement des peintures, jusqu'en 256 , année du remblaiement de l'edifice pour le préserver de l'attaque des Parthes. Onze années étaient certainement insuffisantes pour que l'art de Doura exerce une influence, quelle qu'elle soit. Autant dire que l'analyse des œuvres plus tardies, lorsqu'elle permet d'établir un lien avec des thèmes représentés dans la synagogue, prouve implicitement l'existence de ces thèmes en d'autres œuvres, dont certaines étaient peut-être antérieures à la synagogue et qui son aujourd'hui perdues.

La diffusion de l'iconographie narrative de la bible vers l'Ouest est attestée a partir du $\mathrm{IV}^{\mathbf{e}}$ siècle. C'est la date attribuée aux peintures de la catacombe de la Via Latina à Rome ${ }^{4}$. Catacombe chrétienne, qui se dis-

4 Ferrua A., Le pitture della catacomba di Via Latina. Cité du Vatican 1960. 
tingue des autres catacombes romaines par le nombre important des sujets de l'Ancien Testament qu'elle renferme. De plus, à l'encontre des peintures des autres catacombes, parmi les sujets vétérotestamentaires certains n'ont aucune portée spécifique du point de vue de la doctrine chrétienne, comme Jacob arrivant avec ses fils en Egypte, ou Lot fuyant Sodome avec ses filles. Ces images ont un caractère purement narratif, sans aucun sous-entendu doctrinal. Certaines compositions semblent même être coordonnées à partir d'une succession de scènes individuelles, juxtaposées dans un panneau sans former véritablement un ensemble organique, comme le sacrifice d'Isaac où l'on distingue clairement les trois épisodes du récit. Enfin, plusieurs épisodes son visualisés par des formules déjà attestées à Doura: on peut citer comme exemple le songe de Jacob, Jacob bénissant Ephraïm et Manassé ou Moïse ôtant ses chaussures au Mont Sinaï.

Le célèbre manuscrit, connu sous le nom de Genèse de Cotton, est daté du $v^{e}$ siécle et attribué à Alexandrie. Aujourd'hui intégralement publiée $^{5}$, l'iconographie de ce codex a connue une diffusion considérable. Son influence est attestée dans un grand nombre de manuscrits occidentaux, dont les bibles carolingiennes du scriptorium de Tours et jusqu'à des versions commentées de la bible, à l'usage d'un publique large comme la Genèse de Millstatt du xIVe siècle ${ }^{6}$. En grande partie détruite dans un incendie en 1731, la Genèse de Cotton est surtout connue à travers les mosaïques de la basilique de Saint-Marc de Venise, auxquelles ce manuscrit servit de modèle. Les quelques 300 ilustrations du manuscrit originel ont été intégrées dans le texte, suivant le récit fidèlement, sans que les sujets à portée doctrinale ou les simples éléments narratifs aient été distingués. Ce cycle ne comporte presque aucun détail qui se réfererait à une source littéraire juive, à l'exception des Antiquités Judaïques de Flavius Josèphe, œuvre qui a cependant circulée davantage en milieux chrètiens que juifs. En revanche, le peintre a repris certaines formules de l'art hellénistique en les adaptant au récit biblique. La plus célèbre de ces adaptations est la création d'Adam en trois étapes, visualisée à l'aide de l'iconographie créée pour illustrer la création de l'homme par Prométhée. Cette création de l'homme en trois étapes fut employée durant des siècles et rendue célèbre par les frontispices des bibles de Tours ${ }^{7}$.

\footnotetext{
5 Weitzmann, K., Kesslef, C.H., The Cotton Genesis. British Library, Codex Otho B VI. Princeton 1986

6 Klagenfurt, Museum Rodolfinum, Cod. VI, 19. Cf. KRACHER, A., Millstätter Genesis und Physiologus Handschrift. Graz 1967.

KESSLER, H.L., The llustrated Bibles from Tours. Princeton 1965.
} 
La Genèse de Vienne ${ }^{8}$, du vie siècle, est aujourd'hui attribuée soit à Antioche, soit à la Palestine. Conçue dès le départ d'être une «bible à images", chaque page du manuscrit est divisèe en deux parties, le registre supérieur étant réservé au texte, le registre inférieur aux peintures.

Les images sont composées d'épisodes juxtaposés qui suivent le récit d'événement en événement. Pour maintenir la correspondance entre le texte et les illustrations, le texte est souvent abrégé. Les images, d'une beauté exquise, renferment nombre d'éléments fondés sur la littérature rabbinique et qui ont probablement été repris dans ce manuscrit byzantin d'un modèle juif. Exemples de telles allusions à l'exégèse juive sont "l'homme" que recontre Joseph allant visiter ses frères, évoqué sous les traits d'un ange ${ }^{9}$, ou encore les deux anges qui escortent Jacob rentrant de chez Laban, et qui le prennent sous leur protection dès qu'il franchit les frontières de la Terre sainte ${ }^{10}$.

Au vle siècle encore, fut écrit la Topographie chrétienne de l'alexandrin Cosmas Indicopleustes ${ }^{11}$. Les images concordantes des trois copies conservées de cette œure ${ }^{12}$ font penser que celles-ci remontent à l'original, aujourd'hui perdu. Les illustrations bibliques de la Topographie montrent les mêmes caractéristiques que les peintures de la catacombe de la Via Latina, ou certains panneaux de Doura: une succession d'épisodes juxtaposés dans l'espace réservé, sans syntaxe eta sans coordination. Telle est l'illustration du sacrifice d'Isaac ${ }^{13}$, ou celle de la vocation de Moïse ${ }^{14}$. K. Weitzmann a suggéré qu'il s'agit d'illustrations transférées d'un texte biblique, originaire peut-être d'Antioche ou de Palestine, nécessairement antérieur au vie siècle. Le texte en question aurait pu être un Octateuque, semblable aux quatre manuscrits conservés de cette composition typique de Byzance.

Car c'est également au vle siècle que Kurt Weitzmann ${ }^{15}$ fait remonter l'original de la version illustrée de l'Octateuque, connue de copies du

\footnotetext{
8 Vienne, Nationalbibliothek codex theol. gr. 31. Cf. MAZAL, O., Kommentar zur Wiener Genesis. Frankfurt am Main 1980 et la bibliographie ibidem.

Fol. $15 \mathrm{v}$

10 Fol. $11 \mathrm{r}$

Cf. Wolska- Conus, W., Cosmas Indicopleustès Topographie Chrétienne. Sources Chrétiennes n. ${ }^{\circ} 141,159,197$. París 1963-1973.

12 Vat. gr. 699, IXe s.; Sinaiticus Gr. 1186, Xle.; Florence, Laur. Plut. IX, 28, Xle s.

13 Ms. Sinaiticus 1186 , fol. $98 \mathrm{r}$.

14 Ibidem. fol. $101 \mathrm{v}$.

15 Weitzmann, The frescoes of the Dura Synagogue.
} 
$\mathrm{X}^{\theta}{ }^{16}$ et $\mathrm{du} \mathrm{XII}^{\theta^{17}}$ siècles, et d'un exemplaire fragmentaire du XIIle siècle ${ }^{18}$. On he pent pas sépares siècle. Leurs cycles d'images sont particulièrement riches, allant jusqu'a environ 400 miniatures pour un manuscrit, dont 150 pour le seul texte de la Genèse. Nombre de scènes renferment des éléments ayant leur source dans l'exégèse juive, et plusieurs illustrations reprennent des formules déjà attestées à Doura: le songe de Jacob, le cycle du sacrifice d'Isaac parmi bien d'autres. Alors que la grande majorité des épisodes est présentée selon une formule identique dans toutes les copies, dans certains cas on relève des modifications intervenues entre la copie du $\mathrm{XI}^{\theta}$ siècle et celles $d u \mathrm{XI}^{\ominus}$. Un exemple particulièrement intéréssant est l'illustration de la naissance de Moïse. Dans l'Octateuque du $\mathrm{Xl}^{\theta}$ siècle la scène reprend la formule de Doura, la fille du Pharaon descendant elle-même au fleuve. De la copie du x $\|^{\ominus}$ siècle, ce midrash juif a disparu, la scène au bord du fleuve étant remplacée par une scène de naissance "classique", telle qu'elle fut employée pour illustrer la naissance d'Alexandre le Grand, celle de Jean-Baptiste ou de la Vierge. Certes, l'éloignement progressif du modèle, la perte de l'interprétation littéraire originelle suffisent pour expliquer une telle mutation. On doit néanmoins remarquer que cette mutation est intervenue à une époque de clarification et d'affirmation de certaines positions doctrinales au sein du christianisme.

Dans les œuvres passées en revue, on a constaté la présence de deux traditions. L'une issue d'Antioche, ou peut-être de la Palestine, plus proche de la tradition attestée à Doura et aussi aux textes exégétiques juifs, dont les témoins majeurs sont la Topographie de Cosmas, la Genèse de Vienne et les Octateuques. Le témoin principal de la tradition parallèlle, caractérisé par une influence hellénistique prépondérante, est la Genèse de Cotton. Toutefois, une telle distinction est quelque peu artificielle car il y a des œuvres où les deux tendances sont présentes simultanément. Telles sont les ivoires de l'Antependium de Salerne ${ }^{19}$, œuvres d'un atelier d'Amalfi de la fin $\mathrm{du} \mathrm{xl}^{\mathrm{e}}$ siècle, dont certains éléments montrent des affinités avec le cycle de la Genèse de Cotton, d'autres avec celui des Octateuques.

La diffusion de ces traditions iconographiques vers l'Ouest de l'Europe est attestée à partir du $V I^{e}$ siècle. Le témoin majeur de cette diffusion est un manuscrit particulièrement problématique: le Pentateuque latin connu sous l'appellation peu justifiée de Pentateuque d'Ashburnham ${ }^{20}$. Si la da-

Vatican, Bibl. Apostolique ms. gr. 747.

Ibídem, ms. gr. 746; Istanbul Serail. Smyrne, Ecole évangélique (périt dans un incendie).

18 Mont Athos, Vatopedi Cod. 602.

- Cf. Bergmann, R.P., The Salerno Ivories. Ars Sacra From Medieval Amalfi. Cambridge, Mass.-London 1980.

${ }_{20}$ Paris, Bibl. nat., fr. 2334. Cf. NARKISs, B., "Towards a further Study of the Ashburnham Pentateuch", Cahiers Archéologiques, XIX, 1969, 15-60, et la bibliographie ibidem. 
tation du début du $\mathrm{VII}^{\mathrm{e}}$ siècle a obtenu le consensus de la majorité des historiens, la définition de son lieu d'origine est encore question ouverte. Selon l'une des hypothèses, soutenue par H. Quentin concernant le texte ne pent, être separé ${ }^{21}, W$. Neuss ${ }^{22}$ et J. Domínguez Bordona ${ }^{23}$ pour les illustrations, le Pentateuque serait une œuvre espagnole. Le texte latin suit la version de la Vulgate. Les légendes suscrites aux images reflètent la même version, toutefois, sous l'écriture à l'encre, d'autres sous-titres écrits au crayon dépendent de la Vetus Latina. Le manuscrit, fortement diminué par rapport à son volume originel, comporte actuellement 19 peintures en pleines pages, qui subsistent des 69 peintures originelles que suggèrent les reconstitutions ${ }^{24}$. Ces peintures se présentent comme des mosaïques de scènes individuelles, assemblées sans plan identifiable, sans ordre défini. Dès $1901^{25} \mathrm{~J}$. Strzygowsky a proposé de voir dans ces peintures une transposition d'illustrations fragmentées ayant accompagné un texte. Des éléments stylistiques convergeantes -les costumes, les coiffures, le décor architectural, la faune et la flore, voire certains coutumes (l'enterrement de Jacob) typiques de l'Orient- ont suggéré que le modèle, peut-être sous la forme d'un rouleau illustré, appartenait à la région syro-palestinienne. Des rapporchements avec les costumes et les coiffures féminines représentés sur des tombes palmyréniens - dont certaines apparaissent également dans les peintures de Doura - invitaient à dater ce modèle du Ille-IVe siècle. Plusieurs hébraïsmes dans le texte, un nombre important de thèmes iconographiques fondés sur la littérature rabbinique ${ }^{26}$, la présence de formules déjà attestées à Doura, enfin la lecture de droite à gauche -sens de l'écriture hébraïque ou araméenne- de plusieurs scènes ont amené à formuler l'hypothèse que ce modèle syro-palestinien du \|\|$^{e}-\|\|^{e}$ siécle fut créé pour, et au sein des communautés juives de la région.

Sans pouvoir résoudre le problème de la provenance du Pentateuque, l'analyse de ses peintures permet au moins de conclure à la présence, au début du vlle siècle, en Espagne ou dans une région limitrophe, d'un modèle dont les illustrations étaient disséminées à l'intérieur des colonnes

21 Quentin, H., Mémoire sur l'établissement du texte de la Vulgate. Rome 1922, pp. 414.

22 Neuss, W., Die Katalanische Bibelillustration am Wende des ersten Jahrtrausend und die altspanische Buchmalerei. Leipzig 1922, 59-62.

23 Domínguez- Bordona, J., Spanish Illumination. New York 1948, pp. $5 \mathrm{sg}$.

24 Cf. NARKISS, art. cité dans note 20

${ }_{25}$ Strzygowski, J., Orient oder Rom. Beiträge zur Geschichte der spätantiken und frühchristlichen Kunst. Leipzig 1901.

26 Cf. GutmanN, J., "The Jewish Origin of the Ashburnham Pentateuch", Jewish Quarterly Review, 44, 1953, 55-72; repris dans No Graven Images. New York, éd. J. Gutmann, 1971, pp. 329-346. 
du texte. Ce système, plus archaïque que celui des pleines pages, était aussi celui appliqué dans la Genèse de Cotton, dans les Octateuques, et sans doute dans l'archétype dont dérivent tous les autres témoins, y compris les fresques de Doura-Europos. Ce système est encore attesté dans un groupe de manuscrits bibliques castillans, dont la bible codex 2 de la Collégiale de San Isidoro de Léon, probablement illustrée par le célèbre scribe Florentius et copiée par son disciple Sanctius en $960^{27}$. Une autre bible, également conservée à San Isidoro (codex 3), copiée en 1162 , reprend la mise en page, et la grande majorité des illustrations de la bible de 960, avec quelques variantes toutefois, qui invitent à supposer qu'il s'agit de deux copies indépendantes d'une même modèle. Au même groupe appartient la bible de San Millàn de Cogolla (Madrid, Real Academia de Historia, cod. 2), datée d'environ 1200-1220, dont les illustrations, toujours insérées dans les colonnes d'écriture, dérivent probablement du même modèle. Une autre bible, copiée par le méme Florentius en 943 , conservée autrefois au monastère castillan d'Ona, est mentionnée par l'historien bénédictin du $x \mathrm{~V}_{11 I^{e}} \mathrm{~s}$., Grégorio de Argaiz ${ }^{28}$. Enfin, des rapports de visiteurs $d u X V I I I^{e} \cdot x x^{e}$ siècle ont fait état d'une quatrième bible illustrée à San Isidoro même, manuscrit qui est aujourd'hui perdu et au sujet duquel $\mathrm{J}$. Williams pose la question s'il s'agissait du modèle des bibles de 943 , de 960 et de 1162. Les manuscrits castillans sont exceptionnels par l'abondance des illustrations vétérotestamentaires, par le fait d'avoir conservé le système archaïque d'illustrations fragmentées, hérité des rouleaux illustrés et qui se singularise dans l'art du moyen âge dont la tendance était plutôt de regrouper et coordonner les épisodes individuels en compositions. Ce groupe de manuscrits avait aussi une tradition textuelle particulière, célèbre surtout en raison des gloses extensives de la Vetus Latina souvent inscrites dans les marges. Cette tradition particulière de la Vulgate, dont la récension est attribuée à l'évêque espagnol Peregrinus, du v $v^{e}$ siècle ${ }^{29}$, est présente dans les trois bibles mentionnées. La bible de 960 est considérée par les spécialistes comme en étant le témoin le plus fidèle.

Ce groupe de bibles illustrées se distingue encore par son iconographie. John Williams a montré ${ }^{30}$ l'affinité du frontispice du Lévitique du ms. de 960 -la seule peinture en pleine page-, avec la composition d'une

27 Williams, J., “A Castilian Tradition of Bible Illustration», Journal of the Warburg and Courtauld Institutes 28, 1965, 66-85; idem., “A model for the Leon Bibles", Madrider Mitteilungen, VIII, 1967, pp. 281-286.

${ }_{28}$ Cf. Williams, Castilian Tradition, p. 68.

29 Ibidem, p. 71, n. 25.

so Ibidem, p. 72 ss. 
des fresques de Doura et, d'une façon générale, les connotations juives de la peinture. Ceci invite, de nouveau et à propos d'un autre document, à s'interroger sur l'existence d'illustrations transmises par, ou dérivées de documents juifs. Ou, pour formuler autrement la question: il y a-t-il des indices qui permettent de supposer l'existence, au ve-Vle siècle, en Occident ou à la péninsule ibérique, de textes juifs illustrés.

Si l'on veut limiter l'enquête sur les témoins conservés, la réponse négative sera catégorique. $\mathrm{Si}$, au contraire, on entreprend une investigation en profondeur, en cherchant à décéler le processus dont les témoins conservés ne marquent que l'aboutissement, la réponse devra être beaucoup plus nuancée.

Pour commencer, il convient de passer en revue les documents, qui peuvent être pris en considération pour une telle enquête. Les plus anciens manuscrits hébraïques d'Espagne sont des bibles, et aucun manuscrit de cette région antérieur à la fin du $x \|^{\ominus}$ siècle ne nous est connu. L'ornementation des plus anciens codices est purement graphique et fonctionnelle. Nous avons montré ailleurs l'affinité profonde qui existe entre la décoration fonctionnelle des plus anciennes bibles hébraïques d'Espagne et une tradition ornamentale connue des bibles de Théodulphe, évêque d'Orléans sous Charlemagne, originaire lui-même de la péninsule ${ }^{31}$. Des ornements peints apparaissent seulement vers le milieu du XIIle s. II s'agit de peintures en pleines pages, placées toujours hors texte, soit de pagestapis à décor floral, soit de compositions symboliques à double volet, représentant le mobilier du Sanctuaire. Ces deux types de peintures apparaissent vers la même période, au milieu et au troisième quart du xille siècle, et tous deux à Tolède. Ils ont en commun d'être de pur style oriental, qui se démarque clairement de l'art environnant.

La page-tapis aux double palmettes superposées, reprend un motif attesté depuis le ville siècle sur le pourtour oriental de la Méditerrannée: sur des bois sculptés de la mosquée d'Aqsa, sur les mosaïques de la mosquée Omeyyade de Damas, sur de stucs sculptés de la mosquée de Kairouan, puis, suivant la conquète arabe, ce motif est importé à la péninsule ibérique et apparaît dans le décor architectural de l'Andalousie, et de Tolè$\mathrm{de}^{32}$.

La migration de la double page au mobilier du Sanctuaire ne peut pas être documentée avec autant de précision, car le modèle en était pro-

31 Cf., Sed Rajna, G., "Toledo or Burgos?", Journal of Jewish Art, 2, 1975, pp. 11-13.

32 Ibidem, fig. 15-20. 
bablement dans des manuscrits qui ne sont pas parvenus jusqu'à nous. Le style des peintures: les objets plats, plaqués contre un fond neutre et incolore, l'absence du modelé pour éviter toute illusion de profondeur, l'emploi de l'or comme unique couleur, réduisant la polychromie à un jeu de la lumière et de l'ombre, son autant de traits typiques de l'art du Proche-Orient, repris dans ces bibles sans aucun infléchissement vers les normes de l'art gothique du xille siècle. Le peintre a recopié fidèlement son modèle, sans intervenir pour en adapter le langage formel aux tendances de l'art contemporain. Quant à l'iconographie, elle présente une variante transposée en peinture de la composition symbolique qui est omniprésente dans l'art juif, sur différents médias, depuis les premiers siècles de notre ère. Le rapprochement avec le frontispice du Lévitique de la bible castillane de 960 est parlant. II y a certes, des variantes importantes, notamment la figure d'Aaron dans le codex de Florentius, il y a aussi trois siècles d'écart entre les deux manuscrits. II serait sans doute hasardeux de supposer un lien de dépendance directe entre les bibles castillanes d'une part et les bibles hébraïques d'autre part. Tout au plus peut-on considérer la peinture de la bible hébraïque comme un indice supplémentaire de la présence de modèles juifs dans la péninsule ibérique.

Cette composition symbolique a été reprise dans les bibles hébraïques d'Aragon et de Catalogne pendant plus d'un siècle. Et, hormis celle-ci, aucune illustration proprement dite n'a été admise dans les bibles, à l'exception de quelques cas isolés. Une véritable imagerie biblique surgit seulement vers les années 1310-1320, à la faveur de la création d'un type nouveau de manuscrit: la haggada de Pâques. II s'agit d'un livre peu volumineux, renfermant les prières, les lectures didactiques et les hymnes de louange lus pendant la soirée précédant la fête, au cours de laquelle on commémore la délivrance de l'esclavage et la sortie miraculeuse d'Egypte. Le texte de la haggada, élaboré depuis l'époque de la Mishna, fut fixé vers le milieu du $x \|^{\mathrm{e}}$ siècle et à partir de cette date produit comme livre indépendant. Destiné à l'usage familial, à l'éducation des enfants, ce livre fut souvent embelli d'ornements et aussi profusément illustré. De telles haggadot ont été produites dans la plupart des grands centres de la dispora, en Espagne comme aux pays germaniques et en Italie. Celles provenant de Castille et de Catalogne se distinguent des autres tant par l'organisation du livre que par une iconographie particulière. Nous connaissons six manuscrits de ce type qui ont un intérêt particulier pour le sujet de cet exposé. Tous présentent la même ordonnance: le texte, pourvu d'ornements, comporte des illustrations dans les marges qui visualisent les gestes rituels liés à la fête ou les paraboles didactiques du récit. Ornements, illustrations marginales et écriture sont, dans cette partie du livre, stylistiquement homogènes. Cet ensemble homogène de texte + illustra- 
tions, est précédé d'une série plus ou moins longue d'images bibliques, composées en pleines pages, annexées en tête du volume. Les images sont constituées d'épisodes juxtaposés, répartis en compartiments -deux ou quatre par page - qui visualisent I'histoire biblique dans l'ordre chronologique du récit. II y a des cycles longs, commençant aux premiers chapitres de la Genèse; d'autres sont limités au livre de l'Exode. Le récit est continu, avec quelques interruptions et ommissions, nécéssitées par l'exiguiité de l'espace. Toutefois, la sélection ne dépend pas d'un plan privilégiant les thèmes devenus des hauts lieux de la théologie. II s'agit d'illustrations narratives, probablement détachées de cycles plus longs, qui visualisent fidèlement le texte biblique, ou plus exactement, une version enrichie de légendes de ce texte.

Le critère auquel les historiens de l'art attachent le plus d'importance lors de l'analyse portant sur l'origine d'une iconographie est son rapport au texte dans lequel elle apparait. Si ce rapport est directe, si l'illustration visualise le contenu même du texte et non pas une interprétation qui a pu être surajoutée, on peut admettre que l'illustration fut suscitée par le texte et partant, créée pour et en même temps que celui-ci. Dans le cas contraire, lorsque le rapport entre texte et image n'est pas directe, il est probable que l'iconographie fut créée pour un texte différent et réemployée dans le nouveau contexte.

C'est le cas des cycles bibliques des haggadot Les images bibliques qui les composent se réfèrent à des événements auquel le rituel de la Pâque ne fait aucune allusion. Les protagonistes des épisodes bibliques n'y sont même pas évoqués, les histoires qu'elles visualisent n'ont aucun lien avec le contenu du livre. La dichotomie entre cycles bibliques et illustrations textuelles a souvent marquée aussi le style: celui des images bibliques accusant plus d'une fois un décalage important par rapport au reste du manuscrit.

Il a été souvent proposé de voir dans les psautiers médiévaux les modèles des haggadot d'Espagne. Ceci est sans doute vrai en ce qui concerne l'organisation du livre. Mais c'est le seul point commun entre les deux. Ni l'esprit qui a conçu les deux types d'œuvres, ni le répertoire utilisé de part et d'autre ne relèvent du même courant de pensée. Dans les psautiers, le texte est précédé par une série d'images de l'Ancien et du Nouveau Testament, choisies en fonction de leur teneur doctrinale et structurées en compositions autonomes. Les cycles des haggadot sont, au contraire, construits à partir de scènes individuelles juxtaposées, souvent sans autre coordination que celle imposée par les contraintes de l'espace. La sélection ne reflète aucun plan doctrinal, les scènes théologiquement significatives et des éléments purement narratifs se succèdent 
sans que les premières soient valorisées par rapport aux seconds. C'était précisément le caractère propre aux cycles bibliques des manuscrits de l'Antiquité tardive et des Octateuques. C'est également avec l'icnographie de ces manuscrits anciens que celle des images bibliques des haggadot revèlent le plus d'affinités.

L'analyse exhaustive de l'iconographie des cycles bibliques des six haggadot conservées et la comparaison avec celle des cycles anciens est extrêmement complexe, et dépasse les objectifs fixés à cet exposé. On se limitera par conséquent à citer qualques exemples particulièrement parlants. Nous les choisirons dans les trois haggadot ayant des cycles plus complets, incluant aussi la Genèse: La Haggada d'Or (Brit. Libr. Add. ms 27210) ${ }^{33}$, d'environ 1320; la Haggada catalane (Brit. Libr. Or. ms 2884) ${ }^{34}$ de 1330-1340; la Haggada de Sarajevo (Sarajevo, Musée national) ${ }^{35}$, de 1340-1350, ce dernier ayant le cycle le plus long, qui renferme des illustrations de tout le Pentateuque.

Deux remarques préliminaires permettront de les situer. La première concerne la relation des trois manuscrits entre eux: aucun des trois cycles ne présente la même sélection de scènes, chaque manuscrit renferme des images qui ne se trouvent pas dans les autres. Par conséquent aucun des trois ne peut être considéré comme la copie de l'autre. Les concordances qui existent -et elles sont nombreuses entre la Haggada d'Or et la Haggada catalane - sont le reflet de modèles communs et non pas les indices d'une dépendance directe. La deuxième remarque concerne la relation de la tradition iconographique des haggadot aux traditions des cycles bibliques anciens. L'imagerie des trois haggadot révèle des liens aussi bien avec la tradition des Octateuques qu'avec celle qui caractérise le groupe d'œurres dépendant de la Genèse de Cotton.

Nous choisirons d'abord des exemples du cycle de la Haggadah catalane, qui est le plus instructif pour une telle analyse dans la mesure où la peintre, très maladroit, n'a fait aucune tentative pour adapter son modèle au nouveau contexte, l'iconographie et les articulations originelles des scènes apparaissent donc de manière particulièrement claire. Adam nommant les animaux dans le jardin d'Eden (fol. 1v, b) reprend une iconographie attestée dans les Octateuques (e.g. Vat. gr. 747, fol. 19v). Eve émergeant du côté d'Adam (fol. 2r, a) appartient à la même tradition (Vat.

33 Narkiss, B., The Golden Haggada. London 1970.

34 Narkiss, B., Cohen Mushlin, A., TCherikover, A., lluminated Hebrew Manuscripts in the British Isles. I. London-Jerusalem 1982, n. 12.

35 Ed. Fac-similé, Belgrad-Sarajevo, 1984. 
gr. 746 , fol. 37r), alors que la séquence triple de la tentation est une imagemiroir de celle qui apparait sur l'Antependium de Salerne ${ }^{36}$, qui appartient au groupe des œuvres dérivées de la Genèse de Cotton. De cette même tradition dépend l'épisode de Noé construisant l'arche dont l'iconographie dans la Haggadah catalane (fol. $2 v$, a) est une réplique précise de celle de l'ivoire de Salerne ${ }^{37}$, avec une modification dans le nombre des ouvriers suite à un changement dans les proportions de l'espace disponible. Dans l'épisode de l'ivresse de Noé (fol. $3 r$, a), la forme de la vigne est également typique l'iconographie des œuvres qui dérivent de la Genèse de Cotton: elle apparaît sous la même forme dans les mosaïques de SaintMarc de Venise ${ }^{38}$, dans le manuscrit du XIVe siècle de l'aHistoire Universelle» conservé à Vienne ${ }^{39}$, parmi d'autres ${ }^{40}$. Les éléments iconographiques caractéristiques de la recension de la Genèse de Cotton sont particulièrement nombreux dans le cycle de Jospeh: la forme du puits dans lequel les frères jettent Joseph (fol. $6 \mathrm{v}, \mathrm{b}$ ) ${ }^{41}$; le banquet des frères dans le champ (ibid.,); la séquence au prison avec les deux officiers endormis, puis racontant leur rève à Joseph (fol. $7 \mathrm{v}, \mathrm{a}$ ); la forme particulière des greniers qui ressemblant à des pyramides tronquées, laquelle forme, dans la Genèse de Cotton, n'était que le résultat accidentel d'une peinture rognée par le haut, mais qui fut recopiée telle quelle dans les mosaïques de Saint Marc ${ }^{42}$ ainsi que dans les autres documents auquel ce codex a servi de modèle ${ }^{43}$, et qui est également reprise dans la Haggadah (fol. $8 v$, a).

L'articulation originelle des scènes dans le cycle de la Haggadah catalane demeure nettement perceptible. En effect, le peintre s'est contenté de juxtaposer les scènes d'une même séquence, en les séparant seulement par un arbre indéfiniment répété. La lecture du cycle va de droite à gauche, dans le sens de l'écriture hébraïque et, ce qui est encore plus important, les actions à l'interieur des scènes progressent également de droite à gauche. Enfin plusieurs scènes se réfèrent à des récits midrashiques, telles Abraham jeté à la fournaise ardente sur l'ordre de Nimrod,

\footnotetext{
BERGMAN, Ibidem, fig. 5 .

Ibidem, fig. 8.

Weitzmann-Kessler, The Cotton Genesis, fig. 131.

Vienne, Nat. Bibl., cod. 2567, fol 7v; WeitzMANN-Kessler, fig. 132.

40 Par exemple le Hortus Deliciarum de Herrard de Hohenburg; cf. GREEN, Rosalie B., Herrard of Hohenburg. Hortus Delciarum. Studies of the Warburg Institute, vol. 36. London 1979.

Cf. NARKISS, op. cit., note 33, fig. 165, à comparer avec Weitzmann-Kessler, fig. 349, 350,

42 WeitZMANN-KesSLer, fig. 453.

43 Ibidem, fig. 454.
} $354,357$. 
ou Joseph faisant semblant d'identifier ses frères grâce à une coupe divinatoire au cours du repas qu'il leur a offert.

Le cycle de la Haggadah d'Or concorde sur de nombreux points avec celui de la Haggadah catalane. Mais les différences entre les deux sont significatives. La plupart des scènes de la Haggadah d'or progresse de gauche à droite, dans le sens de l'écriture latine. Les peintures de ce manuscrit son l'oeuvre de deux artistes de talent qui ont eu soin de masquer les articulations originelles et de structurer les scènes individuelles en compositions. Enfin 21 épisodes du cycle de la Haggadah catalane ne figurent pas dans ce manuscrit. Bien que d'une vingtaine d'années antérieure, la Haggadah d'Or n'a pu donc être le modèle de la Haggadah catalane. Vue les convergences nombreuses, il s'agit tout au plus de deux copies indépendantes du même modèle.

Toutefois, il serait probablement plus juste de supposer l'existence de plusieurs modèles. C'est au moins l'hypothèse que suggère l'analyse du troisième manuscrit, celle de la Haggadah de Sarajevo. Son cycle est le plus long, allant de la Création jusqu'à la révélation de la loi au Sinaï. La sélection des épisodes dans le cycle de la Genèse et de l'Exode est différente de celle des autres manuscrits. Sur les 55 épisodes représentés dans le cycle de la Genèse, 19 ne figurent pas dans les autres Haggadot. La récension iconographique de ce manuscrit renvoie plus d'une fois à la tradition prévalante dans les Octateuques et dans la Topographie de Cosmas, voire à la formule qui devait être celle de l'archétype. Telles sont le sacrifice d'Isaac en une séquence de deux épisodes (fol. $7 \mathrm{v}, \mathrm{b}, 8 \mathrm{r}$, a) comme dans le manuscrit de Cosmas ${ }^{44}$ et les Octateuques (Vat. gr. 747, fol. $43 r-v)$; le songe de Jacob avec deux anges seulement et le ciel au sommet de l'échelle (fol. 10r, b), suivi de l'onction de la pierre dressée en autel (ibid; Vat. gr. 747, fol 50r), ou encore la danse de Miriam (fol. $28 \mathrm{r}$, b et Vat. gr. 746 , fol. 194v). Mais cette dépendance de la tradition des Octateuques n'est pas exclusive, certains éléments attestent des affinités avec la Genèse de Vienne, telle l'expulsion du Paradis (fol. 3v, b); d'autres avec la Genèse de Cotton, comme Joseph jeté au puits (fol. $12 r$, a). La peintre de cette Haggadah avait connu et employé la formule de mise en page du "leader s'adressant au peuple" dont Florentius a souvent fait usage dans sa bible de 960 . Enfin, le cycle de cette Haggadah est particulièrement riche en matériel midrashique. (Eve avec le fuseau; Isaac sortant à la rencontre de Rebecca à l'heure de la prière de l'aprèsmidi; le vêtement d'Esaü remis à Jacob par Rebecca; Joseph identifiant 
ses frères à l'aide de la coupe divinatoire; les Israélites sortant d'Egypte la main levée; Pharaoh sauvé par un grâce ultime parce qu'il se repentit, etc.). Cette Haggadah, la plus tardive des trois, est donc sous l'aspect des formules iconographiques plus proche de la tradition qui se reflète dans les documents les plus anciens, et renferme en même temps le plus d'éléments authentiquement juifs quant au contenu des images.

Les Haggadot pourvus de cycles d'images bibliques ont surgi à la fin du XIIle et au début du XIVe siècle en Castille et en Catalogne, à la faveur de la création de ce type de rituel comme manuscrit autonome, à une époque où de tels recueils étaient à la mode et l'art du livre hébreu était en pleine floraison. L'analyse rapide, que nous appronfondirons en d'autres occasions, a montré que ces images reflètent des traditions connues de manuscrits antérieurs aux rituels juifs de six ou sept siècles. II n'y a pas la moindre probabilité en faveur de la supposition que les peintres des Haggadot aient eu une connaissance directe des Octateuques ou de leur archétype, ou des autres manuscrits exécutés en Alexandrie, à Antioche, en Palestine. Ces traditions créées en Orient, devaient par conséquent être disponibles en Espagne même. Leur présence à la péninsule ibérique avait déjà été supposée pour expliquer les images du Pentateuque d'Ashburnham et des bibles castillanes. La pénétration des traditions iconographiques importées d'Orient pouvait se faire par l'Italie du Sud ou l'Afrique du Nord, comme c'était le cas d'œuvres littéraires dont plusieurs furent apportées des centres babyloniens et palestiniens aux communautés d'Espagne. Qu'il y avait des traditions parallèles, parait vraisemblable. Que certaines fûrent transmises à l'intérieur des communautés juives, semble probable. Que leur impacte dépassait les limites de cette communauté est prouvé par les peintures du Pentateuque latin et par les illustrations des bibles de Castille.

L'analyse des documents juifs n'apporte pas de preuve décisive concernant l'origine de l'iconographie de ces manuscrits. Elle fournit tout au plus un argument supplémentaire en faveur de la supposition que ce courant d'illustrations bibliques, issue d'une tradition ancienne et importée d'Orient, était disponible en Castille puis en Catalogne entre la fin du $v l^{\mathbf{e}}$ et le milieu, voire la fin du XIV siècle. 
